\title{
Reliability of Hand-Held Dynamometer for assessing Isometric Lumbar Muscles Strength in Asymptomatic Healthy Population
}

\author{
Fahad Tanveer ${ }^{1}$, Syed Asadullah Arslan ${ }^{2}$, \\ Haider Darain ${ }^{3}$, Ashfaq Ahmad ${ }^{4}$
}

\begin{abstract}
Objective: To determine intra-rater and inter-rater reliability of hand-held dynamometer for assessing isometric lumbar muscle strength in asymptomatic healthy population.

Methods: It was a cross-sectional study conducted at the department of physiotherapy, University of Lahore Teaching Hospital, Lahore, Pakistan, from July 2020 to August 2020 through non probability-purposive sampling technique. Thirty healthy subjects were tested at thirty-degree lumbar flexion and zero-degree lumbar extension positions. Two raters assessed isometric strength of lumbar flexor and extensor muscles, by a hand-held dynamometer. Strength was measured and recorded by each of the two raters and reassessed after a week. Correlation and pairwise comparison were done between readings. ICC values were calculated for the assessment of isometric lumbar muscle strength using handheld dynamometer.

Results: A total of 30 healthy subjects had participated with mean age of $22.84 \pm 1.21$ years, height $174.33 \pm 6.83 \mathrm{~cm}$, weight $68.58 \pm 5.08 \mathrm{~kg}$ and $\mathrm{BMI} 22.52 \pm 0.35$. Findings showed an excellent intra-rater (ICC $2, \mathrm{k}=0.95$ to 0.97 ) and inter-rater (ICC $2, \mathrm{k}=0.94$ to 0.95 ) reliability.

Conclusions: Hand held dynamometer demonstrated an excellent intra- and inter-rater reliability for assessment of isometric lumbar muscles strength of healthy subject at clinical setting as it is simple to use, portable and cost-effective for the precise measurement of lumbar muscles strength.
\end{abstract}

KEYWORDS: Lumbar region, Muscle strength dynamometer, Reliability.

doi: https://doi.org/10.12669/pjms.37.2.3621

How to cite this:

Tanveer F, Arslan SA, Darain H, Ahmad A. Reliability of Hand-Held Dynamometer for assessing Isometric Lumbar Muscles Strength in Asymptomatic Healthy Population. Pak J Med Sci. 2021;37(2):461-465. doi: https://doi.org/10.12669/pjms.37.2.3621

This is an Open Access article distributed under the terms of the Creative Commons Attribution License (http://creativecommons.org/licenses/by/3.0), which permits unrestricted use, distribution, and reproduction in any medium, provided the original work is properly cited.

1. Dr. Fahad Tanveer, PhD (Scholar)

2. Dr. Syed Asadullah Arslan, PhD

3. Dr. Haider Darain, PhD,

Institute of Physical Medicine \& Rehabilitation,

Khyber Medical University, Peshawar, Pakistan.

4. Dr. Ashfaq Ahmad, PhD

1,2,4: University Institute of Physical Therapy,

The University of Lahore,

Lahore, Pakistan.

Correspondence:

Dr. Syed Asadullah Arslan,

Associate Professor,

University Institute of Physical Therapy,

The University of Lahore,

Defense Road,

Lahore, Pakistan.

Email: asadshahgilani@gmail.com

* Received for Publication:

September 6, 2020

* Revision Received:

October 26, 2020

* Accepted for Publication: * December 28, 2020

\section{INTRODUCTION}

Lumbar muscles strength plays a vital role in the physical performance and routine physical activity. These lumbar muscles provide lumbar stability during functional movements, transfer and control. ${ }^{1}$ On the other hand, decreased lumbar strength can trigger the surrounding muscles to become hypertonic ${ }^{2}$ which lead to low back pain ${ }^{3}$ and an estimated risk of injury which eventually impact activities of daily living. ${ }^{4}$ Therefore, the assessment of the lumbar strength can be used to avoid primary and secondary traumas. ${ }^{5}$ At present, different valid methods are being used to test the strength of lumbar muscles. ${ }^{6}$ BieringSorensen test, ${ }^{7}$ is a valid measuring method for the strength of lumbar extensors and a prone 
bridging test which has shown to be accurate to assess the strength of lumbar muscles. ${ }^{4}$

The tools that can be used for measuring muscle strength are, hand held dynamometry (HHD), Isokinetic dynamometers or Manual Muscle Testing. Handheld dynamometer can ensure quantified strength measurement and clinically, it is very effective and efficient tool. ${ }^{8}$ It is also considered as a reliable and valid tool to measure the strength of muscles in the upper and lower extremities. No functional measuring tool for calculating the strength of lumbar flexors and extensors has been reported. There was also no evidence for HHD as an effective tool to reliably measure the strength of lumbar flexors and extensors. Therefore, the aim of this study was to determine intra-rater and inter-rater reliability of hand-held dynamometer for assessing isometric lumbar muscles strength in asymptomatic healthy population.

\section{METHODS}

This cross-sectional study was a part of PhD Physical Therapy project conducted at Department of Physiotherapy, University of Lahore Teaching Hospital, Lahore, Pakistan, from July 2020 to August 2020 by using non probabilitypurposive sampling technique. After approval from the Institutional Review Board (IRB-UOLFAHS/690/2020, Dated: 22-01-2020) of the University of Lahore, this study was registered in the ClinicalTrials.gov (NCT04578587).

Thirty healthy subjects ( 26 males \& 4 females) aged between twenty-one to twenty-five years were recruited after their written informed consent for this study. Subjects with the history of acute, subacute or chronic low back pain, history of trauma, neurological disorders, spinal surgery, lumbar spine pathology were excluded from the study. Micro Force Evaluation and Testing-2 HHD (Hoggan Scientific LLC, Salt Lake City) was tested to determine the protocol's for intra and interrater reliability. Both raters were physiotherapists having clinical experience of more than eight years with expertise in assessing isometric muscles strength using HHD. Both raters were unaware of each other's findings and subjects too were unaware of the results.

Based on the recent evidence, positioning protocol of HHD for lumbar flexor and extensor isometric muscles strength was considered. ${ }^{2}$ The isometric strength of lumbar flexors was assessed in supine position at thirty-degree by placing the dynamometer under the suprasternal notch.
Subjects were asked to put their hands over the opposite acromion processes. The isometric strength of the lumbar extensors was assessed in prone position. The dynamometer was placed at the level of T4 and subjects were asked to put their hands on the forehead. A $10 \mathrm{~cm}$ belt was wrapped just above the lateral malleolus and another belt over the anterior superior iliac spine (ASIS) to prevent lumbar motion while assessing the strength of lumbar flexors whereas belt was wrapped over the posterior superior iliac spine to measure the strength of lumbar extensors. Subjects were instructed to maximally contract the muscle for five seconds. ${ }^{9}$ Peak force value was recorded in newton $(\mathrm{N})$ and converted into torque values $(\mathrm{Nm})$ by multiplying with moment arm length. Moment arm length was measured between sternum and ASIS for lumbar flexors strength whereas for strength of lumbar flexors the distance between T4 and PSIS was considered. Subjects were reassessed after a week while keeping the same HHD protocol.

Data was analyzed by using SPSS 21. Descriptive statistics of age, height, weight and BMI were calculated. Mean, standard deviation, intrarater and inter-rater pairwise comparisons and correlations of rater one and rater two for first and second readings were calculated. The intra-rater and inter-rater reliability were calculated by the use of two-way random effects model with multiple raters/measurements reliability (ICC 2, k), with $95 \%$ confidence interval $(\mathrm{CI}) .{ }^{10}$ The normality of all data was checked using the Shapiro-Wilk test. Statistically, significant value was agreed at the level of $5 \%$.

\section{RESULTS}

Distribution of each measurement performed by the evaluators at the time one, time two, and their variability are shown in Table-I. Correlation and pairwise comparison is shown in Table-II and Table-III. Agreement for inter-rater and intra-rater reliability was classified as excellent for intra-rater

Table-I: Descriptive statistics of age (years), Height $(\mathrm{cm})$, weight $(\mathrm{kg})$ and BMI.

\begin{tabular}{lccccc}
\hline & Mean & S.D & Range & Minimum & Maximum \\
\hline Age (years) & 22.84 & 1.21 & 4 & 21 & 25 \\
Height $(\mathrm{cm})$ & 174.33 & 6.83 & 22.20 & 163.80 & 186.00 \\
Weight $(\mathrm{kg})$ & 68.58 & 5.08 & 17.60 & 60.40 & 78.00 \\
BMI & 22.52 & 0.35 & 1.10 & 22.00 & 23.10 \\
\hline
\end{tabular}

S.D: Standard Deviation, BMI: Body Mass Index. 
Table-II: Intra-Rater pairwise comparison of lumbar flexor and extensor muscle strength of rater 1 and rater 2 for $1^{\text {st }}$ and $2^{\text {nd }}$ reading.

\begin{tabular}{|c|c|c|c|c|c|c|}
\hline Flexor & Mean & S. D & Minimum & Maximum & $r(p$-value $)$ & $t$-test ( $p$-value) \\
\hline Rater $1,1^{\text {st }}$ reading & 142.89 & 8.32 & 128.01 & 156.41 & \multirow{2}{*}{$0.995(<0.001)$} & \multirow{2}{*}{$19.306(<0.001)$} \\
\hline Rater $1,2^{\text {nd }}$ reading & 139.82 & 8.06 & 126.90 & 152.34 & & \\
\hline Rater $2,1^{\text {st }}$ reading & 146.78 & 8.34 & 131.97 & 160.37 & \multirow{2}{*}{$0.996(<0.001)$} & \multirow{2}{*}{$25.795(<0.001)$} \\
\hline Rater $2,2^{\text {nd }}$ reading & 143.40 & 8.36 & 128.22 & 157.62 & & \\
\hline Extensor & Mean & S. D & Minimum & Maximum & $r(p$-value $)$ & t-test ( $p$-value) \\
\hline Rater $1,1^{\text {st }}$ reading & 196.58 & 9.87 & 181.97 & 220.17 & \multirow{2}{*}{$0.998(<0.001)$} & \multirow{2}{*}{$29.701(<0.001)$} \\
\hline Rater $1,2^{\text {nd }}$ reading & 193.40 & 10.04 & 198.93 & 216.63 & & \\
\hline Rater $2,1^{\text {st }}$ reading & 201.05 & 9.69 & 186.81 & 224.59 & \multirow{2}{*}{$0.997(<0.001)$} & \multirow{2}{*}{$24.956(<0.001)$} \\
\hline Rater $2,2^{\text {nd }}$ reading & 197.73 & 9.63 & 182.06 & 220.88 & & \\
\hline
\end{tabular}

S.D: Standard Deviation; r: correlation coefficient, p-value: $\leq 0.001$, t-test: paired sample t-test.

measurements of flexors $(\mathrm{ICC}=0.96)$ and extensors $(\mathrm{ICC}=0.97)$, excellent for inter-rater measurements of flexors $(\mathrm{ICC}=0.94)$ and extensors $(\mathrm{ICC}=0.95)$. Agreement limits for 95\% CI are shown in Table-IV and Table-V. The 95\% CI agreement limits of flexors for the intra-rater measurements were inferior while superior for extensors to those for the interrater measurements.

\section{DISCUSSION}

This study has focused on exploring whether the handheld dynamometer is a reliable tool for assessing the isometric strength of lumbar flexors and extensors. Intra-rater pairwise comparison and correlation of lumbar flexors strength of rater one and rater two for $1^{\text {st }}$ and $2^{\text {nd }}$ reading was $19.306(\mathrm{p}$ $<0.001$ ) and 0.995 ( $\mathrm{p}<0.001$ ) respectively whereas inter-rater pairwise comparison and correlation of lumbar flexors strength of rater one and rater two for $1^{\text {st }}$ and $2^{\text {nd }}$ reading was $-58.400(\mathrm{p}<0.001)$ and 0.999 ( $p<0.001)$. Similarly, inter and intra-rater pairwise comparison and correlation of lumbar extensors strength of rater one and rater two for $1^{\text {st }}$ and $2^{\text {nd }}$ reading was 29.701 ( $\mathrm{p}<0.001), 0.998(\mathrm{p}<0.001)$ and -36.709 ( $p<0.001), 0.998(p<0.001)$. Previously no researcher had demonstrated the inter and intrarater pairwise comparison and correlation to find the strength of lumbar flexors and extensors using HHD. Therefore, this was the first study to show intra-rater pairwise comparison and correlation.

Table-III: Inter-Rater pairwise comparison of lumbar flexor and extensor muscle strength of rater 1 and rater 2 for $1^{\text {st }}$ and $2^{\text {nd }}$ reading.

\begin{tabular}{|c|c|c|c|c|c|c|}
\hline Flexor & Mean & S. D & Minimum & Maximum & $r(p$-value $)$ & t-test ( $p$-value) \\
\hline Rater $1,1^{\text {st }}$ reading & 142.89 & 8.32 & 128.01 & 156.41 & \multirow{2}{*}{$0.999(<0.001)$} & \multirow{2}{*}{$-58.400(<0.001)$} \\
\hline Rater $2,1^{\text {st }}$ reading & 146.78 & 8.34 & 131.97 & 160.37 & & \\
\hline Rater $1,2^{\text {nd }}$ reading & 139.82 & 8.06 & 126.90 & 152.34 & \multirow{2}{*}{$0.990(<0.001)$} & \multirow{2}{*}{$-16.236(<0.001)$} \\
\hline Rater $2,2^{\text {nd }}$ reading & 143.40 & 8.36 & 128.22 & 157.62 & & \\
\hline Extensor & Mean & S. D & Minimum & Maximum & $r$ (p-value) & t-test ( $p$-value) \\
\hline Rater $1,1^{\text {st }}$ reading & 196.58 & 9.87 & 181.97 & 220.17 & \multirow{2}{*}{$0.998(<0.001)$} & \multirow{2}{*}{$-36.709(<0.001)$} \\
\hline Rater $2,1^{\text {st }}$ reading & 201.05 & 9.69 & 186.81 & 224.59 & & \\
\hline Rater $1,2^{\text {nd }}$ reading & 193.40 & 10.04 & 198.93 & 216.63 & \multirow{2}{*}{$0.995(<0.001)$} & \multirow{2}{*}{$-21.544(<0.001)$} \\
\hline Rater $2,2^{\text {nd }}$ reading & 197.73 & 9.63 & 182.06 & 220.88 & & \\
\hline
\end{tabular}

S.D: Standard Deviation; r: correlation coefficient, p-value: $\leq 0.001$, t-test: paired sample t-test. 
Fahad Tanveer et al.

Table-IV: Intra-class Correlation Coefficient for intra-rater reliability.

\begin{tabular}{|c|c|c|c|c|c|}
\hline \multirow{2}{*}{ Reliability } & \multirow{2}{*}{ Flexor } & \multirow{2}{*}{ Intraclass Correlation $^{b}$} & \multicolumn{2}{|c|}{$95 \%$ C.I } & \multirow[t]{2}{*}{$p$-value } \\
\hline & & & Lower Bound & Upper Bound & \\
\hline & Rater $1,1^{\text {st }}$ and $2^{\text {nd }}$ reading & $0.963^{\mathrm{a}}$ & -0.007 & 0.993 & 0.000 \\
\hline & Rater $2,1^{\text {st }}$ and $2^{\text {nd }}$ reading & $0.959^{\mathrm{a}}$ & -0.002 & 0.992 & 0.000 \\
\hline \multirow{4}{*}{ Intra-Rater } & \multirow{2}{*}{ Extensor } & \multirow{2}{*}{ Intraclass Correlation $^{b}$} & \multicolumn{2}{|c|}{$95 \%$ C.I } & $p$-value \\
\hline & & & Lower Bound & Lower Bound & \\
\hline & Rater $1,1^{\text {st }}$ and $2^{\text {nd }}$ reading & $0.974^{\mathrm{a}}$ & 0.025 & 0.995 & 0.000 \\
\hline & Rater $2,1^{\text {st }}$ and $2^{\text {nd }}$ reading & $0.970^{\mathrm{a}}$ & 0.012 & 0.994 & 0.000 \\
\hline
\end{tabular}

Two-way random effects model where both people effects and measures effects are random.

a The estimator is the same, whether the interaction effect is present or not, ${ }^{b}$ Type A intraclass correlation coefficients using an absolute agreement definition, p-value: $\leq 0.001$, C.I: Confidence Interval.

Intra-class correlation coefficient for inter rater reliability of lumbar flexors strength was (ICC=0.94-0.94) similar to the study by Harding AT et al which measured back muscle strength using isokinetic dynamometry in two sessions with an interval of seven days on fifty-two healthy subjects. Analysis was done by using (ICC) and had a reliability of $0.83-0.94^{11}$ A study by A Ilyas et al measured reliability of HHD for assessing isometric shoulder flexor and abductor strength. The HHD showed excellent within day (ICC = 0.99-0.99) and between days (ICC $=0.99-0.99)$ intrarater reliability for shoulder flexion and abduction of both sides. ${ }^{12}$ Another study by Mentiplay BF et al. examined inter-rater and intra-rater reliability of HHD for assessing isometric muscle strength of lower extremity. Thirty asymptomatic subjects were assessed on two sessions. Inter-rater and intra-rater reliability was moderate to excellent for hip and knee (ICCs $\geq 0.70$ ), poor-good for ankle muscles (ICCs $=0.31-0.79) \cdot{ }^{13}$ Intra-class Correlation Coefficient for intra rater reliability of lumbar flexors strength was (ICC $=0.95-0.96$ ) similar to the study of David A. Krause, PT et al and Awatani T et al. which had an excellent reliability (ICC, 0.89$0.95)^{14,15}$ Another study by Ashoi L et al. assessed hip muscle group on seventeen participants. All the measurements were taken by an experienced single rater using HHD with a gap of 30 minutes' interval. Analysis was done by using intra-tester reliability $\left(\mathrm{ICC}_{2,1}\right)$ with a reliability of $0.85-0.92$ for all measurements. ${ }^{16}$ A study by Funny Buckinx et al. measured elbow and ankle muscle groups using HHD with Micro FFT 2 Device. ICC values were $0.60(0.37-0.83)$ for the ankle flexors and $0.85(0.74-$ $0.95)$ for the elbow flexors ${ }^{17}$ whereas inter and intraclass correlation coefficient for inter rater reliability of lumbar extensors strength was (ICC $=0.94-0.95$ )

Table-V: Intra-class Correlation Coefficient for inter-rater reliability.

\begin{tabular}{|c|c|c|c|c|c|}
\hline \multirow{2}{*}{ Reliability } & \multirow{2}{*}{ Flexor } & \multirow{2}{*}{ Intraclass Correlation $^{b}$} & \multicolumn{2}{|c|}{$95 \%$ C.I } & \multirow[t]{2}{*}{$p$-value } \\
\hline & & & Lower Bound & Upper Bound & \\
\hline & Rater 1 and rater $2,1^{\text {st }}$ reading & 0.948 & 0.012 & 0.990 & 0.000 \\
\hline & Rater 1 and rater $2,2^{\text {nd }}$ reading & 0.949 & -0.035 & 0.990 & 0.000 \\
\hline \multirow{4}{*}{ Inter-Rater } & \multirow{2}{*}{ Extensor } & \multirow{2}{*}{ Intraclass Correlation $^{b}$} & \multicolumn{2}{|c|}{$95 \%$ C.I } & p-value \\
\hline & & & Lower Bound & Lower Bound & \\
\hline & Rater 1 and rater $2,1^{\text {st }}$ reading & 0.949 & 0.003 & 0.990 & 0.000 \\
\hline & Rater 1 and rater $2,2^{\text {nd }}$ reading & 0.951 & -0.019 & 0.990 & 0.000 \\
\hline
\end{tabular}

Two-way random effects model where both people effects and measures effects are random.

b Type A intraclass correlation coefficients using an absolute agreement definition;

p-value: $\leq 0.001 ;$ C.I: Confidence Interval. 
and (ICC $=0.97-0.97)$ to $0.62(0.41-0.84)$ for the ankle extensors to 0.87 (0.79-0.96) for the elbow extensors. ${ }^{17}$ A study by Hirano $\mathrm{M}$ et al measured isometric knee extensors strength using HHD on forty-two subjects. Analysis was done by using (ICC 1,1) with reliability of $0.75-0.82$ respectively ${ }^{18,19}$ Another study Tudini F described the reliability of HHD for cervical muscles in healthy adults.

Both intra-rater and inter-rater reliability were excellent (ICCs $=0.88$ - 0.97) similar to that of our study..$^{20} \mathrm{~A}$ study by Tarca BD investigated the reliability of HHD for assessing isometric abdominal flexion strength. 35 participants were recruited for test-test reliability on subsequent days. HHD showed good agreement $(\mathrm{ICC}=0.82$ ) and good consistency (ICC $=0.87) .{ }^{21}$ It was observed that all HHD assessments are highly reliable. Thus, $\mathrm{HHD}$ is considered suitable for testing the isometric strength of lumbar muscles in clinical setting.

Limitations of the study: The participants were only tested to measure the isometric strength of lumbar flexors and extensors. Asymptomatic participants were tested by HHD, thus the findings from this study cannot be extrapolated to lumbar symptomatic population.

\section{CONCLUSION}

Based on the results of this study, HHD is a reliable tool for assessing isometric lumbar muscles strength. In a clinical setting, it is viewed as an easy to use, portable and cost-effective for the precise measurement of lumbar muscles strength.

\section{Grant Support \& Financial Disclosures: None.}

Conflict of Interest: None.

\section{REFERENCES}

1. Schuermans J, Van Tiggelen D, Palmans T, Danneels L, Witvrouw E. Deviating running kinematics and hamstring injury susceptibility in male soccer players: Cause or consequence? Gait \& Posture. 2017;57:270-277. doi: 10.1016/j.gaitpost.2017.06.268

2. De Blaiser C, De Ridder R, Willems T, Danneels L, Roosen P. Reliability \& validity of trunk flexor and trunk extensor strength measurements using handheld dynamometry in a healthy athletic population. Phys Ther Sport. 2018;34:180-186. doi: 10.1016/j.ptsp.2018.10.005

3. Ireland ML, Bolgla LA, Noehren B. Gender differences in core strength and lower extremity function during static and dynamic single-leg squat tests. ACL Inj Female Athlete: Springer; 2018. 239-257. doi: 10.1007/9783-662-56558-2_13

4. De Blaiser C, De Ridder R, Willems T, Danneels L, Vanden Bossche L, Palmans T, et al. Evaluating abdominal core muscle fatigue: Assessment of the validity and reliability of the prone bridging test. Scan J Med Sci in Sports. 2018;28:391-399.

5. Mendonca LDM, Bittencourt NF, Ocarino JM, Fonseca ST. P12: Good lumbopelvic stabilization is associated with patellar tendinopathy absence in athletes. Online J Rur Nurs \& Health Care. 2017;17:11-18.

6. De Blaiser C, De Ridder R, Willems T, Vanden Bossche L, Danneels L, Roosen P. Impaired core stability as a risk factor for the development of lower extremity overuse injuries: A prospective cohort study. The Amer J Sports Med. 2019;47:1713-1721. doi: 10.1177/0363546519837724
7. Read PJ, Oliver JL, Croix MBDS, Myer GD, Lloyd RS. A review of fieldbased assessments of neuromuscular control and their utility in male youth soccer players. J Strength and Cond Research. 2019;33:283-288. doi: $10.1519 /$ JSC 0000000000002069

8. Sung K-S, Yi YG, Shin H-I. Reliability and validity of knee extensor strength measurements using a portable dynamometer anchoring system in a supine position. BMC MSK Disorders. 2019;20:320-324. doi: 10.1186/ s12891-019-2703-0

9. McKay MJ, Baldwin JN, Ferreira P, Simic M, Vanicek N, Burns J, et al. Normative reference values for strength and flexibility of 1,000 children and adults. Neurology. 2017;88:36-43. doi: 10.1212/WNL.0000000000003466

10. Koo TK, Li MY. A guideline of selecting and reporting intraclass correlation coefficients for reliability research. J Chiropractic Med. 2016;15:155163. doi: $10.1016 /$ j.jcm.2016.02.012

11. Harding AT, Weeks BK, Horan SA, Little A, Watson SL, Beck BR. Validity and test-retest reliability of a novel simple back extensor muscle strength test. SAGE Open Med. 2017;5:20-26. doi: 10.1177/2050312116688842

12. Ilyas A, Ahmad A, Gillani SA, Bukhari B, Gillani H. The Validity and Reliability of a Sphygmomanometer in Assessing Isometric Muscle Strength of Shoulder Flexors and Abductors. Ann King Edward Med Univ. 2019;25:1-6.

13. Mentiplay BF, Perraton LG, Bower KJ, Adair B, Pua Y-H, Williams GP, et al. Assessment of Lower Limb Muscle Strength and Power Using HandHeld and Fixed Dynamometry: A Reliability and Validity Study. Plos One. 2015;10:1-18. doi: 10.1371/journal.pone.0140822

14. Krause DA, Hansen KA, Hastreiter MJ, Kuhn TN, Peichel ML, Hollman JH. A Comparison of Various Cervical Muscle Strength Testing Methods Using a Handheld Dynamometer. Sports Health. 2019;11:59-63. doi: $10.1177 / 1941738118812767$

15. Awatani T, Morikita I, Shinohara J, Mori S, Nariai M, Tatsumi Y, et al. In tra-and inter-rater reliability of isometric shoulder extensor and interna rotator strength measurements performed using a hand-held dynamometer. J Physical Therapy Sci. 2016;28:3054-3059. doi: 10.1589/jpts.28.3054

16. Ishoi L, Holmich P, Thorborg K. Measures of hip muscle strength and rate of force development using a fixated handheld dynamometer: intra-tester intra-day reliability of a clinical set-up. Int J Sports Phys Ther. 2019;14:715-719. doi: 10.26603/ijspt20190715

17. Buckinx F, Croisier JL, Reginster JY, Dardenne N, Beaudart C, Slomian J et al. Reliability of muscle strength measures obtained with a hand-held dynamometer in an elderly population. Clin Physio and Func Imaging. 2017;37:332-340. doi: 10.1111/cpf.12300

18. Hirano M, Katoh M, Gomi M, Arai S. Validity and reliability of isometric knee extension muscle strength measurements using a belt-stabilized hand-held dynamometer: a comparison with the measurement using an isokinetic dynamometer in a sitting posture. J Physical Therapy Sci. 2020;32:120-124. doi: $10.1589 /$ jpts.32.120

19. Chamorro C, De la Fuente C, Rubio J, Campos C, Chirosa LJ. Absolute reliability and concurrent validity of a novel electromechanical pulley dynamometer for measuring shoulder rotation isometric strength in asymptomatic subjects. Study conducted at Pontificia Universidad Católica, Santiago, Chile. Innovation. 2019;69:1000-1005.

20. Tudini F, Myers B, Bohannon R. Reliability and validity of measurements of cervical retraction strength obtained with a hand-held dynamometer. J Manual Mani Ther. 2019;27:222-228. doi: 10.1080/10669817.2019.1586167

21. Tarca BD, Wycherley TP, Meade A, Bennett P, Ferrar KE. Validity and Reliability of Hand-Held Dynamometry for Abdominal Flexion Muscular Assessment. J Sport Rehab. 2020;11:1-4. doi: 10.1123/jsr.2019-0521

\section{Author's Contribution}

FT \& SAA: conceived the idea, designed and are accountable for the accuracy or integrity of the work.

FT, SAA \& AA: data collection, and manuscript writing.

SAA \& HD: statistical analysis, and edited manuscript.

SAA, HD, AA: reviewed and finally approved manuscript. 